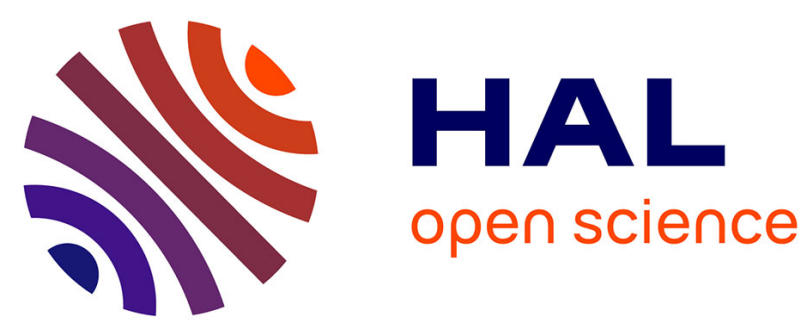

\title{
Incidence du syndrome du canal carpien opéré et poids de l'activité professionnelle dans sa survenue chez les femmes du Maine-et-Loire en 2002-2003
}

Natacha Fouquet, C Ha, Yves Roquelaure, G Raimbeau, Marcel Goldberg, Ellen Imbernon

\section{To cite this version:}

Natacha Fouquet, C Ha, Yves Roquelaure, G Raimbeau, Marcel Goldberg, et al.. Incidence du syndrome du canal carpien opéré et poids de l'activité professionnelle dans sa survenue chez les femmes du Maine-et-Loire en 2002-2003. Congrès international d'épidémiologie Adelf-Epiter, Sep 2008, Paris, France. 56 (5S), pp.327, 2008, Revue d'épidémiologie et de santé publique. 10.1016/j.respe.2008.06.234 . hal-02923891

\section{HAL Id: hal-02923891 \\ https://hal.science/hal-02923891}

Submitted on 2 Sep 2020

HAL is a multi-disciplinary open access archive for the deposit and dissemination of scientific research documents, whether they are published or not. The documents may come from teaching and research institutions in France or abroad, or from public or private research centers.
L'archive ouverte pluridisciplinaire HAL, est destinée au dépôt et à la diffusion de documents scientifiques de niveau recherche, publiés ou non, émanant des établissements d'enseignement et de recherche français ou étrangers, des laboratoires publics ou privés. 


\section{Incidence du syndrome du canal carpien opéré et poids de l'activité professionnelle dans sa survenue chez les femmes du Maine-et-Loire en 2002-2003}

N. Fouquet ${ }^{1,2}$, C. $\mathrm{Ha}^{1}$, Y. Roquelaure ${ }^{2}$, G. Raimbeau ${ }^{2}$, M. Goldberg ${ }^{1}$ E. Imbernon

$1 /$ Institut de veille sanitaire, Saint-Maurice, France ; 2 / Laboratoire d'ergonomie et d'épidémiologie en santé au travail Unité associée InVS - Upres EA 4336, IFR 132, Université d'Angers, Faculté de médecine, France

\section{Contexte}

Le syndrome du canal carpien (SCC) traduit la compression du nerf médian lors de son passage sous le ligament annulaire antérieur du carpe, à la base de la paume de la main. Dans les statistiques de maladies professionnelles indemnisables (MPI), le SCC est le trouble musculosquelettique (TMS) le plus fréquent. En 2004, il constituait 38 \% des MPI au titre du tableau 57 du régime général de Sécurité sociale, devant les pathologies de l'épaule et du coude [1]. Les facteurs de risque professionnels du SCC sont bien établis : mouvements répétitifs du membre supérieur, mouvements de torsion du poignet, utilisation de la pince pouce-index, utilisation d'un outil vibrant, d'un clavier informatique [2,3].

Le Département santé travail de l'InVS a mis en place en 2002 un programme de surveillance épidémiologique des TMS, choisissant la région des Pays-de-la-Loire pour la mise en œuvre d'un réseau pilote. Ce programme vise à constituer un observatoire des TMS d'origine professionnelle. Ses principaux objectifs sont de décrire la fréquence et l'évolution des principaux TMS et des conditions de travail qui leur sont associées, leur distribution par profession et secteur d'activité, d'estimer la part des TMS attribuables à l'activité professionnelle et d'explorer l'utilisation de données médico-administratives à des fins de surveillance épidémiologique.

\section{Objectifs}

Parmi les femmes de 20 à 59 ans du Maine-et-Loire :

estimer l'incidence du SCC opéré en 2002-2003

estimer la part de l'activité professionnelle dans la survenue du SCC.

\section{Méthode}

En collaboration avec les chirurgiens de la main, les habitants du Maine-et-Loire de 20 à 59 ans opérés d'un SCC en 2002-2003 ont été repérés grâce aux données médicales des cliniques pratiquant cette intervention.

L'incidence du SCC a été calculée avec les données du recensement du Maine-et-Loire de 1999. La contribution des facteurs professionnels (secteur d'activité, profession) à la survenue $d u$ SCC a été quantifiée par deux indicateurs :

- la fraction de risque attribuable de SCC dans la population (Frap) à une profession ou un secteur. La Frap au fait de travailler dans un secteur d'activité ou une profession représente la proportion des cas observés dans l'ensemble de la population qui serait évitée si le secteur ou la profession ne présentait pas un excès de risque :

la fraction de risque attribuable chez les exposés (Frae). La Frae représente quant à elle la proportion de cas de SCC qu'on peut attribuer spécifiquement au fait de travailler dans un secteur d'activité ou d'exercer une profession parmi les cas qui surviennent dans ce secteur ou cette profession.

Bien que l'étude aie été menée sur l'ensemble de la population, seuls les résultats portant sur les femmes sont présentés ici, du fait du faible nombre de cas de SCC chez les hommes (447 sur les 1500 cas notifiés).

\section{Résultats}

\section{L'INCIDENCE SELON L'ÂGE}

1053 femmes (70,2 \% des cas) ont été opérées du SCC dans le Maine-et-Loire en 2002-2003, soit une incidence annuelle de 2,7\%. L'incidence augmentait régulièrement avec l'âge (figure 1).

FIGURE 1 INCIDENCE DU SCC CHEZ LES FEMMES SELON L'ÂGE

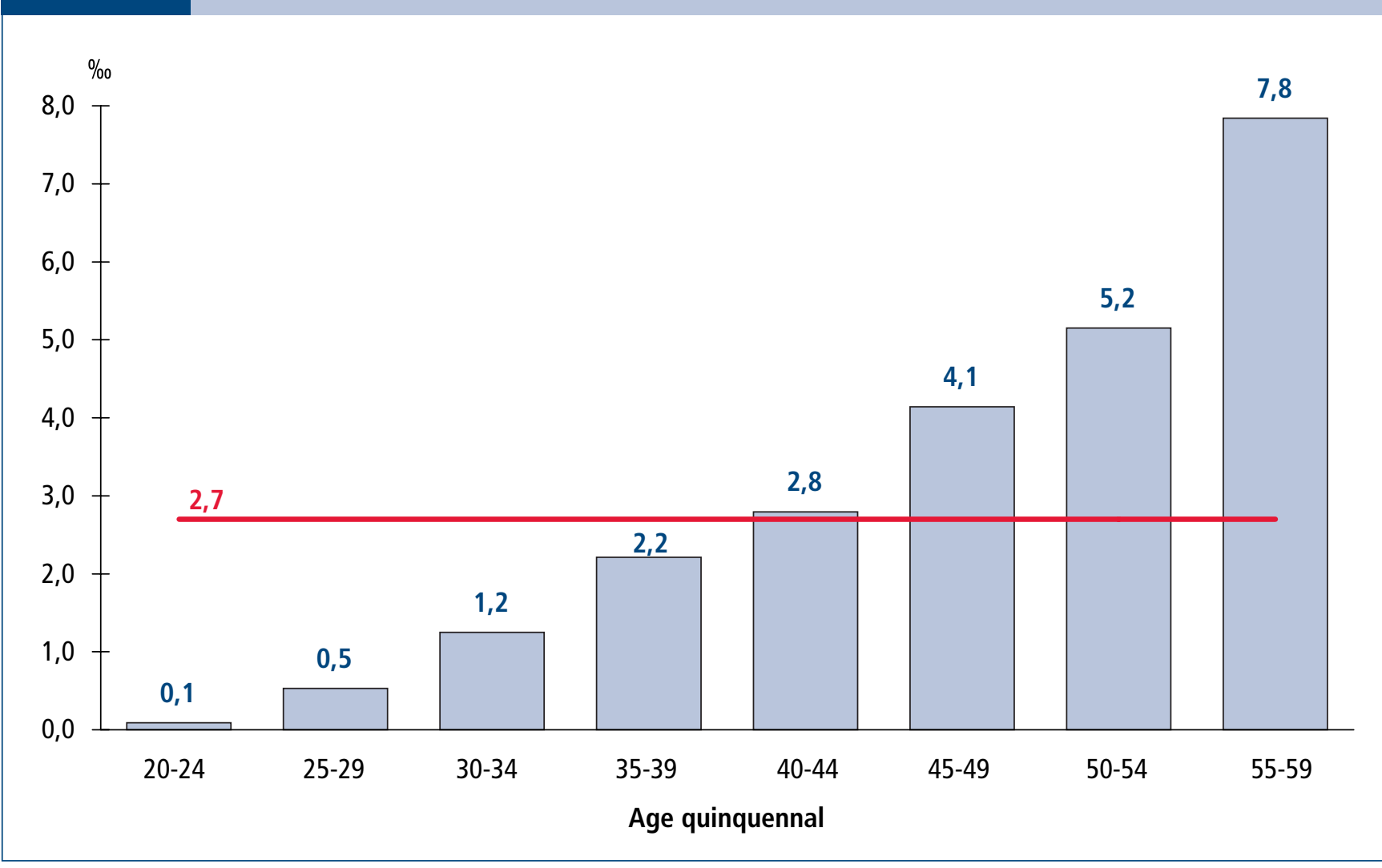

\section{L'INCIDENCE ET LE POIDS DE LA PROFESSION}

Les caractéristiques professionnelles (secteur d'activité et profession) étaient disponibles pour 941 femmes.

Concernant les professions, les incidences les plus élevées s'observaient pour les ouvrières et les professions du tertiaire (fonction publique, commerce, services aux particuliers).

Chez les ouvrières non qualifiées de l'industrie, la Frap était de $11 \%$ (tableau). En d'autres termes, $11 \%$ des cas de SCC opéré seraient évités dans la population active féminine âgée de 20 à 59 ans si cette catégorie professionnelle ne présentait pas d'excès de risque de SCC. Pour toutes les autres professions à risque élevé de SCC, la Frap était plus faible. La Frae variait entre $23 \%$ pour les employées de la fonction publique et $74 \%$ pour les ouvrières agricoles. En d'autres termes, $74 \%$ des cas de SCC opéré seraient évités parmi les ouvrières agricoles âgées de 20 à 59 ans si cette catégorie professionnelle ne présentait pas d'excès de risque de SCC.

\begin{tabular}{|c|c|c|}
\hline $\begin{array}{l}\text { FRACTIONS DE RISQUE ATTRIBUABLE POUR LES } \\
\text { PRÉSENTANT UN EXCĖS DE RISQUE DE SCC CHEZ }\end{array}$ & $\begin{array}{l}\text { DROFESSIONS } \\
\text { Z LES FEMMES }\end{array}$ & \\
\hline Professions & Frap (\%) & Frae (\%) \\
\hline Employées & $6[1-10]$ & 16 [5-26] \\
\hline $\begin{array}{l}\text { Employées du commerce } \\
\text { (vendeuses, caissières) }\end{array}$ & $3[2-5]$ & 46 [27-60] \\
\hline $\begin{array}{l}\text { Personnels des services directs aux particuliers } \\
\text { (serveuses, employées de l'hôtellerie, coiffeuses, } \\
\text { assistantes maternelles, femmes de ménage...) }\end{array}$ & 3 [1-5] & 29 [14-41] \\
\hline $\begin{array}{l}\text { Employées civiles et agents de service } \\
\text { (agents de bureau, agents de service hospitalier, } \\
\text { aides-soignants...) }\end{array}$ & 3 [1-5] & 23 [7-35] \\
\hline Ouvrières & 15 [12-18] & 58 [52-64] \\
\hline $\begin{array}{l}\text { Ouvrières non qualifiées de l'industrie } \\
\text { (agroalimentaire, électricité et électronique, travail du cuir) }\end{array}$ & 11 [9-13] & 69 [64-74] \\
\hline $\begin{array}{l}\text { Ouvrières agricoles } \\
\text { (viticulture, arboriculture fruitière, maraîchage et horticulture) }\end{array}$ & 4 [3-6] & 74 [65-80] \\
\hline $\begin{array}{l}\text { Ouvrières non qualifiées de type artisanal } \\
\text { (nettoyeuses) }\end{array}$ & $2[0-3]$ & 50 [26-66] \\
\hline
\end{tabular}

\section{L'INCIDENCE SELON LE SECTEUR D'ACTIVITÉ}

Les plus fortes incidences étaient observées dans l'agriculture, l'industrie, la santé et l'action sociale, les transports et les services personnels (figure 2).

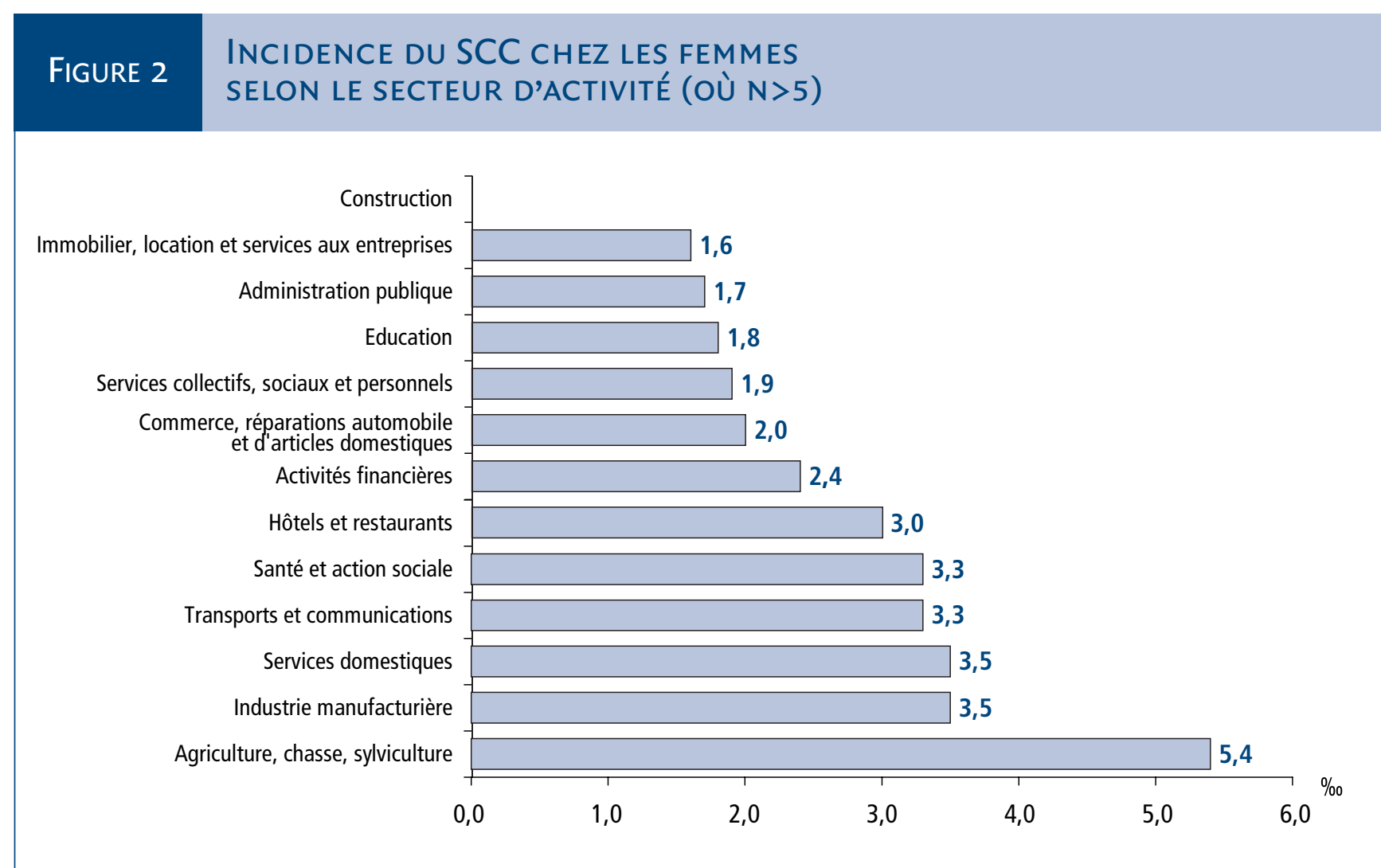

\section{Conclusion et perspectives}

Les professions à risque élevé de SCC sont majoritairement celles exposant à des tâches répétitives et pénibles. Bien que non exhaustif, le recrutement s'est effectué dans les deux cliniques du département les plus importantes en terme de chirurgie du SCC. Le travail se poursuit afin de prendre en compte dans le calcul des fractions attribuables les principaux facteurs de risque individuels de SCC (obésité, diabète, hypothyroïdie).

\section{Références}

[1] Caisse nationale de l'assurance maladie des travailleurs salariés (2004). Statistiques nationales des accidents du travail, des accidents de trajet et des maladies professionnelles (année 2002), Paris.

[2] Bernard BP. (1997) Musculoskeletal disorders and workplace factors. A critical review of epidemiologic evidence for work-related musculoskeletal disorders of the neck, upper-extremity, and low back. Cincinnati, DHHS (NIOSH) publication.

[3] Viikari-Juntura E, Silverstein B. (1999). Role of physical load factors in carpal tunnel syndrome. Scand J Work Environ Health, 25:163-85

Dossier thématique TMS de I'InVS : http://www.invs.sante.fr/surveillance/tms/default.htm 